\title{
Variability in a Large Sample of Sun-Like Stars
}

\author{
Francis C. Fekel and Gregory W. Henry \\ Tennessee State University, Center of Excellence \\ in Information Systems, Nashville, Tennessee 37203, USA
}

Sallie L. Baliunas and Robert A. Donahue

Center for Astrophysics, 60 Garden St. Cambridge, MA 02138, USA

\begin{abstract}
We summarize photometric, spectrophotometric, and spectroscopic results for a sample of nearly 350 nearby late-F to early-K dwarfs and subgiants of various ages. The sun's position in this stellar sample is examined.
\end{abstract}

\section{The Sample}

Our sample consists of nearly 350 single, late-F to early-K dwarfs and some subgiants. The vast majority of stars have $V$ magnitudes brighter than 8.0 and are thus within $50 \mathrm{pc}$ of the sun. The objects have declinations between $75^{\circ}$ and $-15^{\circ}$. Stars from the Ca II H and K list of Wilson (1978), in moving groups such as 61 Cyg, HR 1614, and Wolf 630, and from the Hyades cluster were the first to be included in our sample. Three years ago the number of stars was nearly doubled with the inclusion of objects from the planetary-search sample of Marcy and colleagues, stars from the northern Ca II H and $\mathrm{K}$ survey of Soderblom, and young sun-like stars from Gaidos et al. (2000).

\section{Observations}

Three types of observations have been obtained. Differential photometry has been collected at Fairborn Observatory, Washington Camp, AZ. The program stars fill the menus of four $0.8 \mathrm{~m}$ Automatic Photoelectric Telescopes (APTs) and a number of young, active stars are on the $0.4 \mathrm{~m} \mathrm{APT}$. These telescopes have been built and maintained by Lou Boyd (see http://schwab.tsuniv.edu for more information). In our sample, 81 stars have been observed for 8-13 years, 99 stars for 4-7, years, and 169 stars for 2-3 years. Generally, Strömgren $b$ and $y$ differential magnitudes are obtained for groups consisting of one program star and three comparison stars. Finding "constant" comparison stars is a seemingly never ending challange.

Brightness variations are followed by computing two values. The standard deviation of a single night from the seasonal mean is $\sigma_{\text {short }}$, which is typically about 0.0012 mag for constant pairs. It tracks stellar rotation and active region growth and decay. The standard deviation of the seasonal means from the 
long-term mean has a precision of 0.0001 mag. These values show long period variations associated with solar-like activity cycles.

The Ca II H and K spectrophotometry has been obtained at Mt. Wilson Observatory for several decades (Baliunas et al. 1998). Measurements are made of the S-value, the emission flux of the $\mathrm{Ca}$ II $\mathrm{H}$ and $\mathrm{K}$ lines, which is a proxy for magnetic activity. The ratio of the $\mathrm{H}$ and $\mathrm{K}$ fluxes to the bolometric flux, $R_{\mathrm{HK}}^{\prime}$, is used to examine magnetic variations and cycles in these stars, to measure rotation periods, and to determine an age calibration from cluster and moving

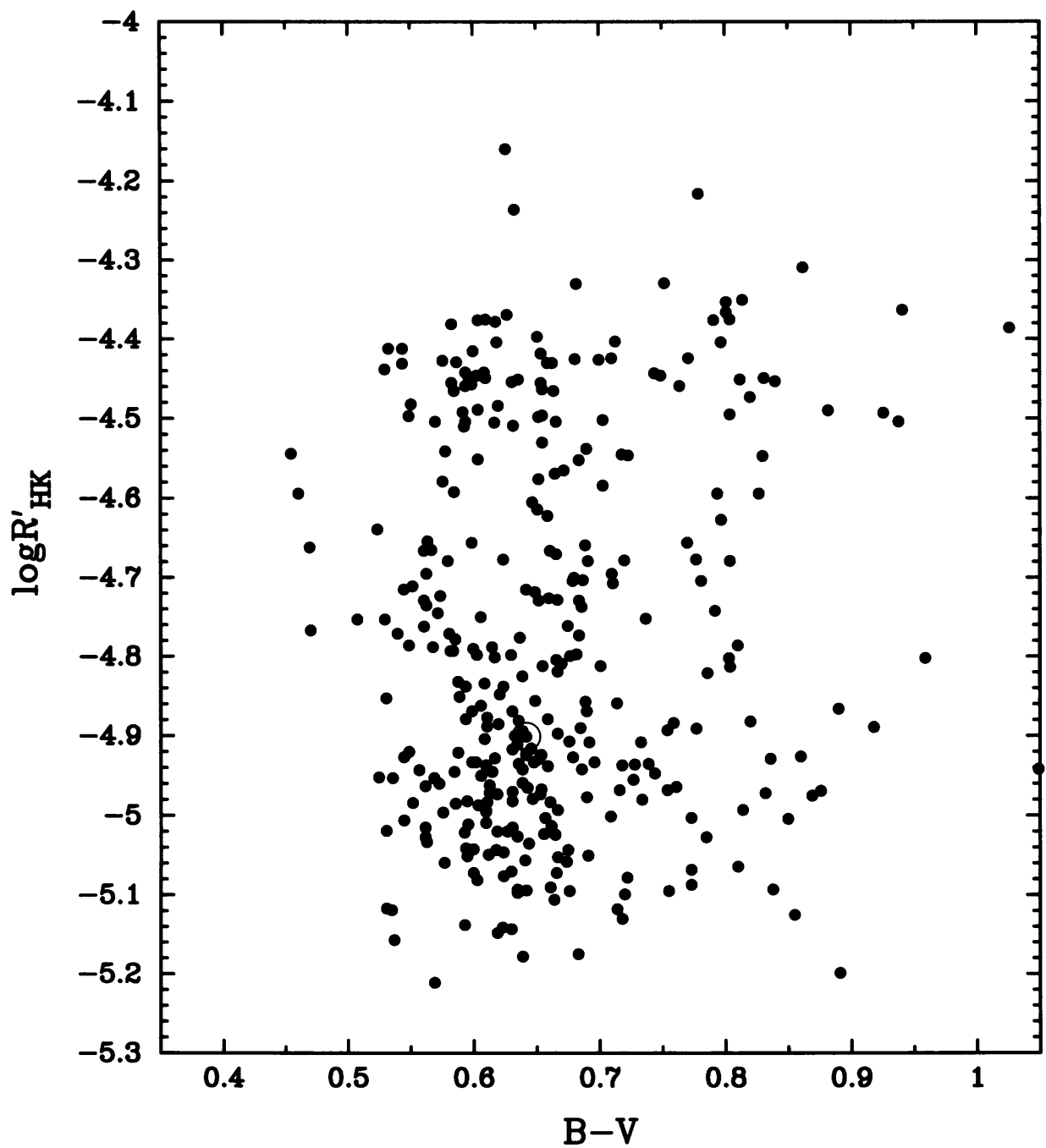

Figure 1. A plot of $\log R_{\mathrm{HK}}^{\prime}$ versus $B-V$ for our sample of stars. The sun is plotted as a circled dot at $\log R_{\mathrm{HK}}^{\prime}=-4.9$. Activity decreases and age increases from top to bottom. 
group stars. The magnetic activity distribution of our stars is shown versus $B-V$ in Fig. 1 .

The high-resolution spectroscopy was collected at Kitt Peak National Observatory with the coudé feed telescope. The spectra have a resolution of about 30,000 at $6430 \AA$. These observations are used to determine spectral types, measure radial velocities good to $\pm 0.3 \mathrm{~km} \mathrm{~s}^{-1}$, obtain $v \sin i$ values with a precision of $1 \mathrm{~km} \mathrm{~s}^{-1}$, and estimate iron abundances.

\section{Questions Posed}

The photometric time series for $25-50 \%$ of our stars is now long enough to provide preliminary answers to some of the following questions. (1) How does solar variability compare to a large sample of similar stars? (2) Are there correlations or anti-correlations between brightness variations and magnetic activity? (3) At what activity level do solar-type stars change from being spot dominated to being plage dominated? (4) Can Maunder minimum stars be identified in our sample? (5) Is there a significant dependence of stellar brightness variations on stellar inclination?

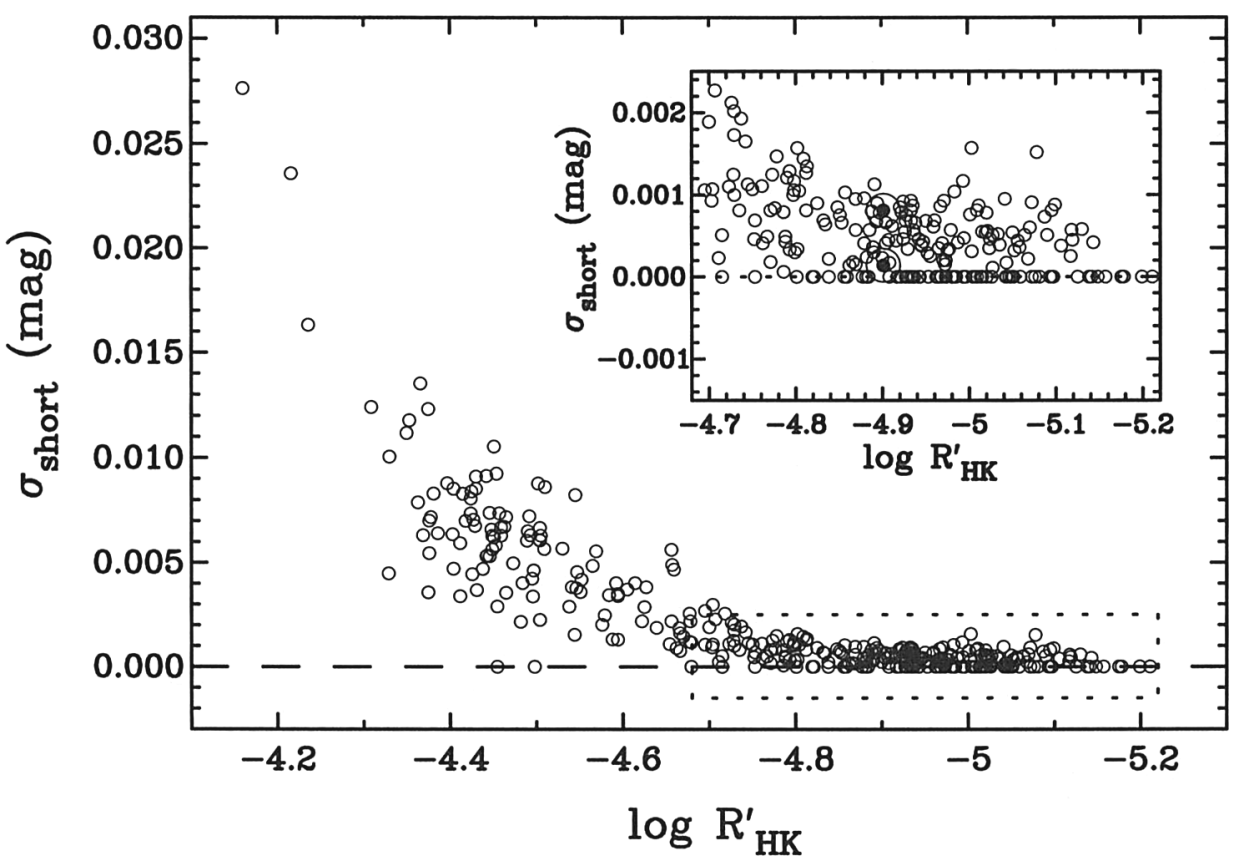

Figure 2. Average short-term light variability versus $\log R_{\mathrm{HK}}^{\prime}$. The inset expands the low-activity region. A circled dot indicates the sun at solar maximum and also at solar minimum. 


\section{Some Preliminary Answers}

(1) Baliunas et al. (1998) and others have shown that in solar-like stars Ca II $\mathrm{H}$ and $\mathrm{K}$ emission decreases with age. Figure 2 plots mean values of shortterm light variability versus mean $\mathrm{HK}$ emission flux. As expected for solar-type stars, light variability decreases with decreasing emission flux and thus, with increasing age. The inset in Figure 2 expands the low-activity region. A circled dot indicates the sun at solar maximum and also at solar minimum. The mean of those two extremes is roughly in the middle of the stellar distribution, indicating that the sun's activity is typical of similar aged solar-like stars.

(2) Radick et al. (1998) made an initial search for correlations or anti-correlations between brightness variations and magnetic activity in 35 solar-like stars. Lockwood et al. (2003) and Radick et al. (2003) have recently updated this work. Our more extensive data set will greatly expand the number of systems that can be examined.

(3) The activity level at which solar-type stars change from being spot dominated to being plage dominated remains to be determined.

(4) On the sun, a period of extremely low solar activity, called the Maunder minimum, occurred in the years before 1700. To search for similar epochs in solar-type stars, one can look for stars that are believed to be significantly younger than the sun but that have an activity level similar to or less than the sun's. For single, solar-type dwarfs, a high $v \sin i$ value is indicative of youth. In Figure 3 we plot our $v \sin i$ values versus $\log R_{\mathrm{HK}}^{\prime}$. For comparison, the sun with a rotation velocity of $2 \mathrm{~km} \mathrm{~s}^{-1}$ has $\log R_{\mathrm{HK}}^{\prime}=-4.9$. On the left side of the figure, rapidly rotating stars are to be expected since the activity

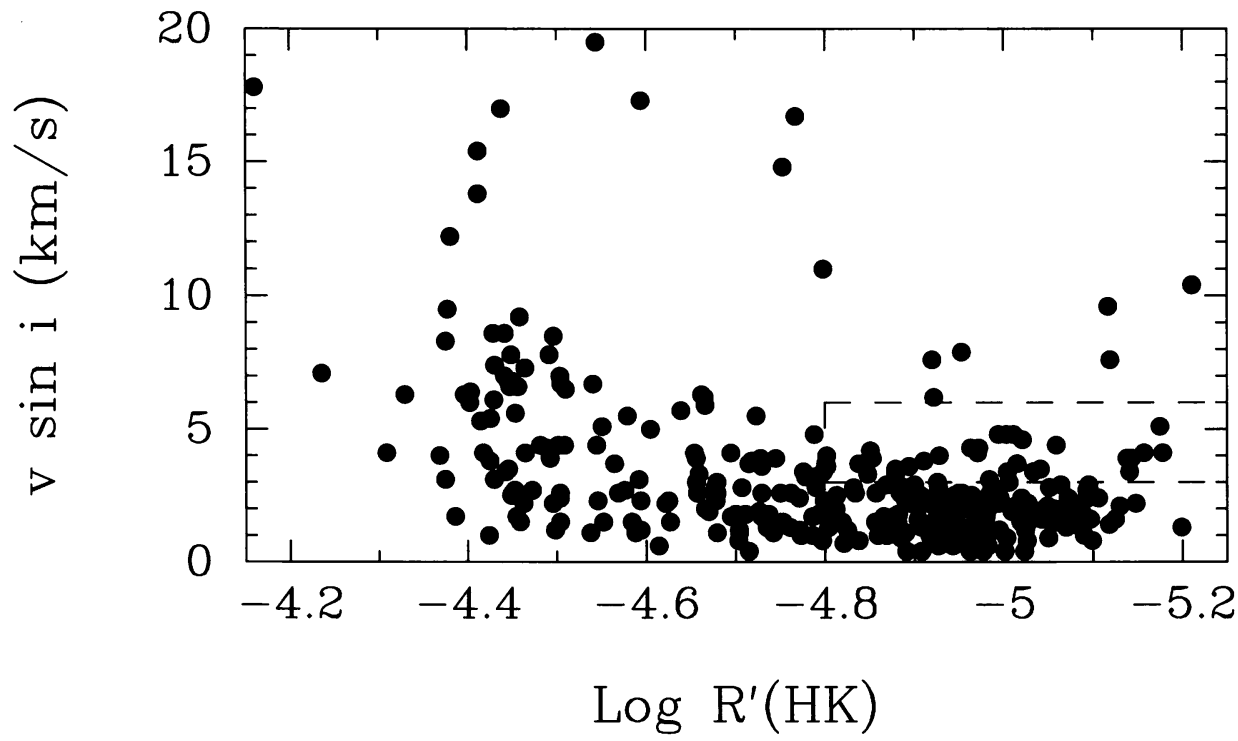

Figure 3. A plot of $v \sin i$ versus $\log R_{\mathrm{HK}}^{\prime}$. The dashed rectangle is the most likely region in which to find Maunder minimum stars. 
level of the stars is high. On the right side of the diagram, the stars with $v \sin i$ $>6 \mathrm{~km} \mathrm{~s}^{-1}$ are $\mathrm{F}$ stars and would be expected to rotate rapidly. The dashed box indicates the most likely region in which to find Maunder minimum candidates, stars with $v \sin i$ values of $3-6 \mathrm{~km} \mathrm{~s}^{-1}$ and $\log R_{\mathrm{HK}}^{\prime}$ values similar or less than that of the sun. Several stars in this region are late- $G$ or early-K dwarfs, and thus, are candidates for being in a Maunder minimum state.

(5) Schatten (1993) made initial predictions concerning the dependence of stellar brightness variations on stellar inclination. The more recent work of Knaack et al. (2001) has predicted a substantially reduced effect. We have not yet begun to compare our results with their prediction.

\section{References}

Baliunas, S. L, Donahue, R. A., Soon, W., \& Henry, G. W. 1998, in Cool Stars, Stellar Systems, and the Sun, Tenth Cambridge Workshop, ed. R. A. Donahue and J. A. Bookbinder (San Francisco: ASP), 153

Gaidos, E. J., Henry, G. W., \& Henry S. M. 2000, AJ, 120, 1006

Lockwood, G. W., Radick, R. R., Henry, G. W., \& Baliunas, S. L. 2003, BAAS, 35,745

Knaack, R., Fligge, M., Solanki, S. K., \& Unruh, Y. C. 2001, A\&A, 372, 1080

Radick, R. R., Lockwood, G. W., Skiff, B. A., \& Baliunas, S. L. 1998, ApJ, 118, 239

Radick, R. R., Lockwood, G. W., Henry, G. W., \& Baliunas, S. L. 2004, in Stars as Suns: Activity, Evolution, \& Planets, IAU Symp. 219, ed. A. K. Dupree and A. O. Benz (San Francisco: ASP), 264

Schatten, K. H. 1993, J. Geophys. Research, 98, 18910

Wilson, O. C. 1978, ApJ, 226, 379 

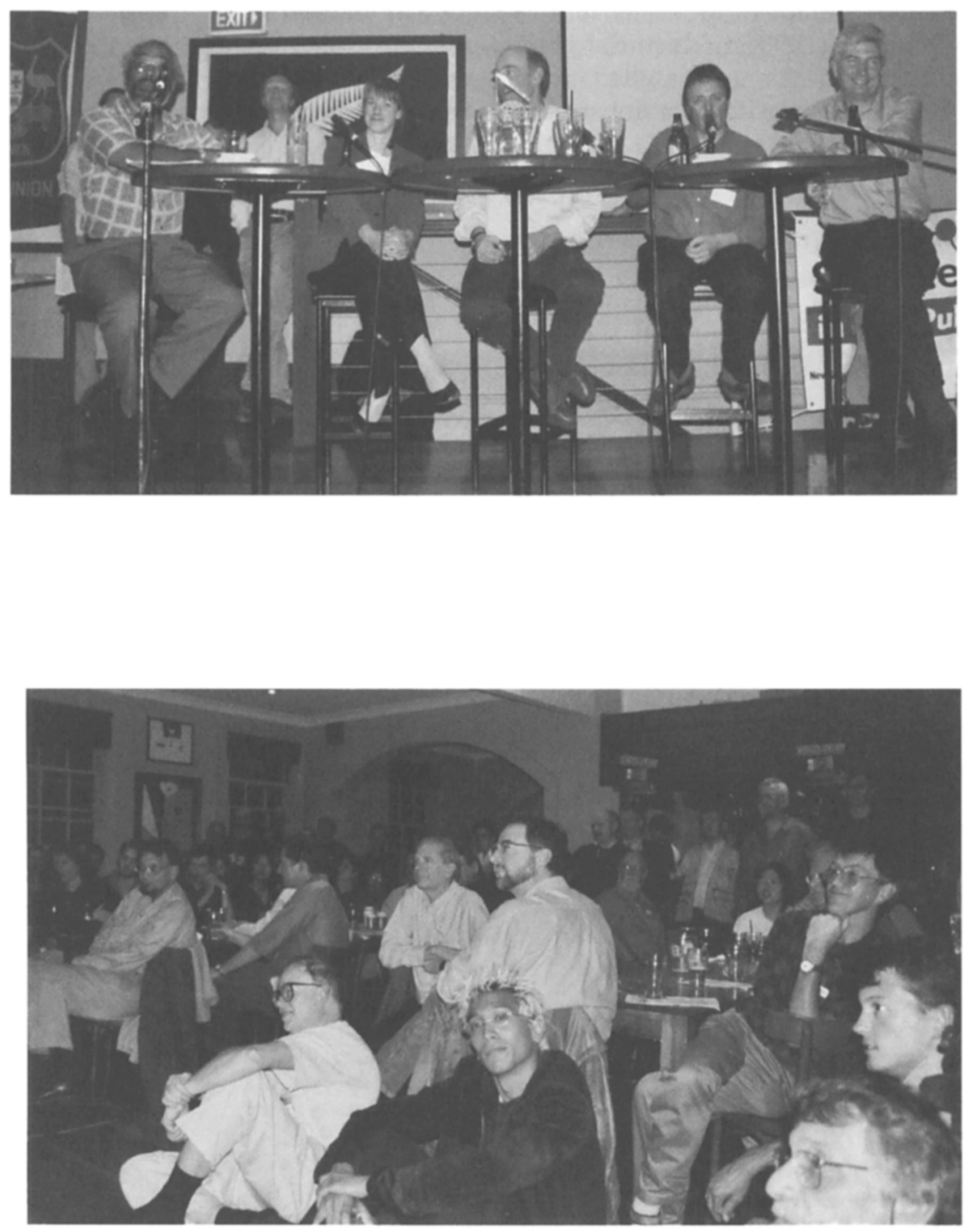